\title{
Comparison between newly diagnosed hypertension in diabetes and newly diagnosed diabetes in hypertension
}

\author{
Chang-Yuan Liu' ${ }^{1}$ Wei Zhang ${ }^{1}$, Li-Nong Ji ${ }^{2}$, Ji-Guang Wang ${ }^{1 *}$ and for the ATTEND investigators
}

\begin{abstract}
Background: Hypertension and diabetes mellitus are often jointly present, especially in early onset cases of either disease. We investigated clinical characteristics of hypertensive patients with newly diagnosed diabetes and diabetic patients with newly diagnosed hypertension.

Methods: Our study subjects were recruited in a China nationwide multicenter registry of hypertension and diabetes $(n=2510)$. We performed logistic regression to compare patients seen for hypertension in cardiology, with newly diagnosed diabetes $(n=137)$ and patients seen for diabetes mellitus in endocrinology, with newly diagnosed hypertension $(n=155)$. Albuminuria was defined as a urinary albumin-to-creatinine ratio of $\geq 30 \mathrm{mg} / \mathrm{g}$, and left ventricular hypertrophy according to the Cornell product index.
\end{abstract}

Results: These two groups of patients with both hypertension and diabetes mellitus were similar in most of the characteristics $(P \geq 0.06)$. However, hypertensive patients with newly diagnosed diabetes, compared to diabetic patients with newly diagnosed hypertension, had a significantly greater body mass index $\left(26.3 \mathrm{vs} .25 .4 \mathrm{~kg} / \mathrm{m}^{2}, P=0.03\right)$ and slower heart rate (73.7 vs. 78.1 beats/min, $P=0.01$ ). In logistic regression analyses adjusted for sex ( $48.3 \%$ women) and age (mean $60.0 \pm 11.5$ years), the odds ratio for newly diagnosed diabetes mellitus versus newly diagnosed hypertension was $1.27(95 \% \mathrm{Cl} 1.03-1.56)$ and $0.80(95 \% \mathrm{Cl} 0.66-0.96)$ for body mass index $\left(+3 \mathrm{~kg} / \mathrm{m}^{2}\right)$ and heart rate (+ 10 beat/min), respectively. Hypertensive patients with newly diagnosed diabetes also had a lower prevalence of albuminuria ( $16.0 \%$ vs. $30.1 \%, P=0.02)$ and slightly and non-significantly higher prevalence of left ventricular hypertrophy $(5.1 \%$ vs. $1.9 \%, P=0.14)$ than diabetic patients with newly diagnosed hypertension.

Conclusions: Earlier or later onset of hypertension than diabetes mellitus may have different risk factors and organ damage.

Keywords: Hypertension, Diabetes mellitus, Body mass index, Heart rate, Albuminuria, Left ventricular hypertrophy

\section{Background}

Hypertension and diabetes mellitus are closely related [1]. These two disorders are often jointly present, and the joint presence of the two disorders confers high risks of target organ damage $[2,3]$ and cardiovascular morbidity

\footnotetext{
*Correspondence: jiguangwang@aim.com

${ }^{1}$ Centre for Epidemiological Studies and Clinical Trials, Shanghai Key Laboratory of Hypertension, The Shanghai Institute of Hypertension, Department of Hypertension, Ruijin Hospital, Shanghai Jiaotong University School of Medicine, Ruijin 2nd Road 197, Shanghai 200025, China

Full list of author information is available at the end of the article
}

and mortality [4]. In a recent China nationwide registry, we found that the prevalence of both hypertension and diabetes mellitus was disproportionate between hypertensive patients seen in cardiology and diabetic patients seen in endocrinology, being $32.9 \%$ and $58.9 \%$, respectively [5]. Because the prevalence of hypertension in the general population was higher than that of diabetes mellitus, one possible explanation could be that only a fraction of hypertension is related to diabetes mellitus, for instance, the so-called obesity-associated hypertension. Nonetheless, other reasons may also play a part. These two disorders may share similar pathogenic pathways 
or one causes another. Diabetes mellitus increases arterial stiffness and in turn systolic pressure and pulse pressure. In addition, the use of some antihypertensive drugs, such as the combination of high-dose thiazides and $\beta$-blockers, [6] is associated with an increased risk of diabetes mellitus. Some antidiabetic drugs may induce sodium and fluid retention, [7] which may also cause a rise in blood pressure. It is therefore intriguing to investigate which group of hypertensive patients would develop diabetes mellitus and which group of diabetic patients would develop hypertension.

Our China nationwide registry in the settings of cardiology and endocrinology offers a unique opportunity to investigate the newly diagnosed hypertension in diabetes and the newly diagnosed diabetes mellitus in hypertension [5]. In the present analysis, we compared the clinical characteristics between newly diagnosed hypertension in diabetes mellitus and newly diagnosed diabetes mellitus in hypertension.

\section{Methods}

\section{Study participants}

Our study participants were recruited from the abovementioned China multicentre registry, which was a cross-sectional study carried out in the departments of cardiovascular and endocrine medicine of hospitals from June 2011 to March 2012 [5]. The study protocol was described in detail previously [5]. In brief, we registered consecutive patients with previously diagnosed hypertension from the departments of cardiovascular medicine and those with previously diagnosed diabetes mellitus from the departments of endocrine medicine. The ethics committees of all participating hospitals approved the study protocol. All subjects gave written informed consent.

To be eligible for inclusion, a patient had to be at least 20 years old, and was able to attend two clinic visits 2 to 5 days apart. At the first clinic visit, physicians administered a standardized questionnaire to collect information on medical history, lifestyle, and use of medications. Blood pressure and anthropometry were measured. At the second clinic visit, blood pressure was measured for the second time. Venous blood samples were drawn after overnight fasting for measurements of plasma glucose, glycosylated haemoglobin A1 (HbA1c) and serum lipids. Morning void urine samples were collected for urinary measurements. Oral glucose tolerance test (OGTT) was performed in the hypertensive patients without known diabetes mellitus [5]. We excluded pregnant women, patients with a history of type 1 diabetes mellitus, or patients participating in another study in the past 3 months.
A total of 2510 patients were registered from 20 departments of cardiology $(n=1330)$ and 20 departments of endocrinology $(n=1180)$ of 25 hospitals [5]. The present analysis, however, was restricted to 137 hypertensive patients seen in cardiology with newly diagnosed diabetes mellitus by an oral glucose tolerance test (OGTT) and to 155 diabetic patients seen in endocrinology with newly diagnosed hypertension according to blood pressure on two clinic visits.

\section{Clinic blood pressure and OGTT}

Blood pressure was measured using the validated Omron HEM-7201 automated oscillometric blood pressure monitor (Omron Healthcare, Kyoto, Japan) at the first and second clinic visits. On each of the two occasions, three consecutive blood pressure readings were obtained in the seated position after the subjects had rested for at least $5 \mathrm{~min}$. These six readings on two clinic visits were averaged for statistical analysis in all subjects and for the diagnosis of hypertension in patients seen in endocrinology without known hypertension. The newly diagnosed hypertension was defined as a blood pressure of at least $140 \mathrm{mmHg}$ systolic or $90 \mathrm{mmHg}$ diastolic.

In patients seen in cardiology without previously diagnosed diabetes mellitus, OGTT was performed for the diagnosis of diabetes mellitus, which was defined as a plasma glucose concentration of at least $7.0 \mathrm{mmol} / \mathrm{L}$ at fasting or $11.1 \mathrm{mmol} / \mathrm{L} 2 \mathrm{~h}$ after ingestion of $75 \mathrm{~g}$ of glucose dissolved in water.

\section{Electrocardiogram (ECG) and urinary measurements}

Standard 12-lead electrocardiogram (ECG) was recorded in all subjects. ECG left ventricular hypertrophy was defined according to the Cornell product index as $(\mathrm{RaVL}+\mathrm{SV} 3) \times \mathrm{QRS}$ duration $>244 \mathrm{mV} \cdot \mathrm{ms}[8]$.

Urinary routine test was performed on fresh urine samples at the laboratory of each participating hospitals. Urinary albumin and creatinine excretions were measured using the immunochemical method in a core laboratory certified by the College of American Pathologists (https ://www.cap.org). In the absence of apparent urological infections on urine samples, albuminuria was defined as a urinary albumin-to-creatinine ratio more than $30 \mathrm{mg} / \mathrm{g}$. Albuminuria included both microalbuminuria (30$299 \mathrm{mg} / \mathrm{g}$ ) and macroalbuminuria ( $\geq 300 \mathrm{mg} / \mathrm{g}$ ).

\section{Other measurements}

Anthropometric measurements included body weight, body height, and waist and hip circumferences. Body mass index was calculated as the body weight in kilograms divided by the body height in meters squared. Overweight was defined as a body mass index of 24.0 to 
$27.9 \mathrm{~kg} / \mathrm{m}^{2}$, and obesity as a body mass index of $28 \mathrm{~kg} / \mathrm{m}^{2}$ or greater. Central obesity was defined as a waist circumference of $\geq 90 \mathrm{~cm}$ for men and $\geq 85 \mathrm{~cm}$ for women.

Dyslipidemia was defined as a serum triglycerides concentration of $1.70 \mathrm{mmol} / \mathrm{L}$ or higher, a serum total cholesterol concentration of $5.18 \mathrm{mmol} / \mathrm{L}$ or higher, a serum low-density lipoprotein (LDL) cholesterol concentration of $3.37 \mathrm{mmol} / \mathrm{L}$ or higher, or a serum high-density lipoprotein (HDL) cholesterol concentration of $1.04 \mathrm{mmol} / \mathrm{L}$ or lower, or as the use of statin or other lipid lowering agents [9].

Ischaemic heart disease included myocardial infarction and angina. Ischaemic heart disease and stroke were self-reported.

\section{Statistical analysis}

For database management and statistical analysis, we used SAS software (version 9.2, SAS Institute, Cary, NC, USA). Means and proportions were compared by the Student t-test and Fisher's exact test, respectively. Continuous measurements with a skewed distribution were normalized by logarithmic transformation and represented by geometric mean and $95 \%$ confidence interval.
Logistic regression analyses were performed to study the associations of interest.

\section{Results}

Demographics of the study population

Table 1 shows the demographics of 137 patients who were seen for hypertension in cardiology and had newly diagnosed diabetes mellitus and 155 patients who were seen for diabetes mellitus in endocrinology and had newly diagnosed hypertension. Hypertensive patients with newly diagnosed diabetes mellitus, compared to diabetic patients with newly diagnosed hypertension, had a significantly $(P \leq 0.03)$ lower proportion of women (40.9\% vs $54.8 \%)$ and were slightly older ( $2.4 \pm 0.7$ years).

\section{Clinical characteristics of newly diagnosed diabetes and newly diagnosed hypertension}

Hypertensive patients with newly diagnosed diabetes mellitus, compared to diabetic patients with newly diagnosed hypertension, had significantly $(P \leq 0.0001)$ lower plasma fasting glucose and HbA1c. However, they had similar systolic and diastolic blood pressure $(P \geq 0.47)$. Among the other clinical characteristics, statistical

Table 1 Characteristics of hypertensive patients with newly diagnosed diabetes mellitus and diabetic patients with newly diagnosed hypertension

\begin{tabular}{|c|c|c|c|}
\hline Characteristic & $\begin{array}{l}\text { Newly diagnosed diabetes } \\
\text { mellitus }(n=137)\end{array}$ & $\begin{array}{l}\text { Newly diagnosed hypertension } \\
(n=155)\end{array}$ & $P$ value \\
\hline Men, n (\%) & $56(40.9)$ & $85(54.8)$ & 0.02 \\
\hline Age, years & $60.0 \pm 11.5$ & $57.6 \pm 10.8$ & 0.06 \\
\hline Body mass index, $\mathrm{kg} / \mathrm{m}^{2}$ & $26.3 \pm 3.7$ & $25.4 \pm 3.5$ & 0.03 \\
\hline Obesity, n (\%) & $43(31.4)$ & $31(20.0)$ & 0.03 \\
\hline Waist circumference, $\mathrm{cm}$ & $92.1 \pm 16.7$ & $90.2 \pm 10.2$ & 0.26 \\
\hline Central obesity, n (\%) & $50(52.1)$ & $46(47.9)$ & 0.22 \\
\hline Current smoking, n (\%) & $22(16.1)$ & $32(20.6)$ & 0.31 \\
\hline Alcohol intake, n (\%) & $20(14.6)$ & $36(23.2)$ & 0.06 \\
\hline Systolic blood pressure, mmHg & $144.2 \pm 16.9$ & $145.6 \pm 15.1$ & 0.47 \\
\hline Diastolic blood pressure, $\mathrm{mmHg}$ & $84.2 \pm 12.5$ & $83.7 \pm 10.7$ & 0.73 \\
\hline Heart rate, beats/min & $73.7 \pm 12.7$ & $78.1 \pm 13.6$ & 0.01 \\
\hline Plasma fasting glucose, $\mathrm{mmol} / \mathrm{l}$ & $6.78(5.86-7.88)$ & $8.00(6.66-10.86)$ & $<0.0001$ \\
\hline Plasma glycosylated haemoglobin A1c, \% & $6.71 \pm 0.98$ & $7.99 \pm 1.97$ & $<0.0001$ \\
\hline Serum triglycerides, $\mathrm{mmol} / \mathrm{l}$ & $1.52(1.03-2.29)$ & $1.34(0.90-2.25)$ & 0.73 \\
\hline Serum total cholesterol, mmol/l & $4.98 \pm 1.09$ & $5.06 \pm 1.18$ & 0.57 \\
\hline Serum HDL cholesterol, mmol/l & $1.30 \pm 0.34$ & $1.36 \pm 0.42$ & 0.25 \\
\hline Serum LDL cholesterol, mmol/l & $3.01 \pm 0.99$ & $3.05 \pm 0.96$ & 0.73 \\
\hline Ischaemic heart disease, n (\%) & $23(16.8)$ & $10(6.5)$ & 0.01 \\
\hline Myocardial infarction, n (\%) & $4(2.9)$ & $1(0.6)$ & 0.14 \\
\hline Stroke, n (\%) & $9(6.6)$ & $5(3.2)$ & 0.18 \\
\hline
\end{tabular}

Values are mean \pm standard deviation, median (interquartile range) or number of subjects (\%). For the definitions of obesity, central obesity, ischaemic heart disease, myocardial infarction and stroke, see "Methods"

$H D L$ high-density lipoprotein, LDL low-density lipoprotein 
significance $(P \leq 0.03)$ was observed for body mass index and heart rate.

Further categorical analyses (Fig. 1) according to body mass index (normal weight, overweight and obesity) and heart rate $(<60,60-90$, and $\geq 90$ beats $/ \mathrm{min})$ showed that the percentage of hypertensive patients with newly diagnosed diabetes mellitus increased from $40.0 \%$ in normal weight subjects to $45.8 \%$ and to $58.1 \%$ in overweight and obese subjects, respectively ( $P$ for trend was 0.02 ) and decreased from $55.9 \%$ in patients with a heart rate below 60 beats $/ \mathrm{min}$ to $47.6 \%$ and $38.0 \%$ in patients with a heart rate of $60-89$ beats $/ \mathrm{min}$ and $\geq 90$ beat $/ \mathrm{min}$, respectively ( $P$ for trend was 0.10 ).

In sex and age-adjusted multiple logistic regression analyses, the odds ratio for newly diagnosed diabetes mellitus versus newly diagnosed hypertension was 1.27 for $3.0 \mathrm{~kg} / \mathrm{m}^{2}$ greater body mass index (95\% CI $1.03-1.56$, $P=0.03)$ and 0.80 for 10 beats $/ \mathrm{min}$ faster heart rate $(95 \%$ CI $0.66-0.96, P=0.02$, Table 2).

\section{Risk of albuminuria, left ventricular hypertrophy and ischaemic heart disease}

Hypertensive patients with newly diagnosed diabetes mellitus, compared to diabetic patients with newly diagnosed hypertension, had a significantly lower prevalence of albuminuria $(16.0 \%$ vs. $30.1 \%, P=0.02)$, higher prevalence of ischaemic heart disease $(16.8 \% \mathrm{vs}$. $6.5 \%, P<0.01)$ and slightly and non-significantly higher prevalence of ECG-left ventricular hypertrophy $(5.1 \%$ vs. $1.9 \%, P=0.14$, Fig. 2 ).
Table 2 Odds ratio for newly diagnosed diabetes mellitus in hypertension $(n=137)$ versus newly diagnosed hypertension in diabetes $(n=155)$

\begin{tabular}{lll}
\hline Variable & $\begin{array}{l}\text { Odds ratio (95\% } \\
\text { confidence interval) }\end{array}$ & P value \\
\hline Age $(+10$ years) & $1.24(0.99-1.56)$ & 0.06 \\
Male sex & $1.75(1.08-2.84)$ & 0.02 \\
Body mass index $\left(+3 \mathrm{~kg} / \mathrm{m}^{2}\right)$ & $1.27(1.03-1.56)$ & 0.03 \\
Heart rate $(+10$ beats $/ \mathrm{min})$ & $0.80(0.66-0.96)$ & 0.02
\end{tabular}

In a stepwise multiple logistic regression model, we forced age and sex and considered body mass index, waist-to-hip ratio, current smoking and alcohol intake, heart rate, serum triglycerides, and serum total and high-density cholesterol for entry and stay at a significance level of $P \leq 0.10$

After adjustment for sex, age, body height and body weight, current smoking and alcohol intake, mean arterial pressure, plasma glucose and serum triglycerides and total to HDL cholesterol ratio, the odds ratio in newly diagnosed diabetes mellitus versus newly diagnosed hypertension was 2.02 (95\% CI $0.46-8.77$, $P=0.35)$ for ECG left ventricular hypertrophy, 3.03 (95\% CI 1.23-7.47, $P=0.02$ ) for ischaemic heart disease and $0.53(95 \% \mathrm{CI} 0.29-0.96, P=0.03)$ for albuminuria.

\section{Discussion}

Our finding was that hypertensive patients with newly diagnosed diabetes mellitus had a greater body mass index and higher prevalence of left ventricular hypertrophy and ischaemic heart disease and a slower resting
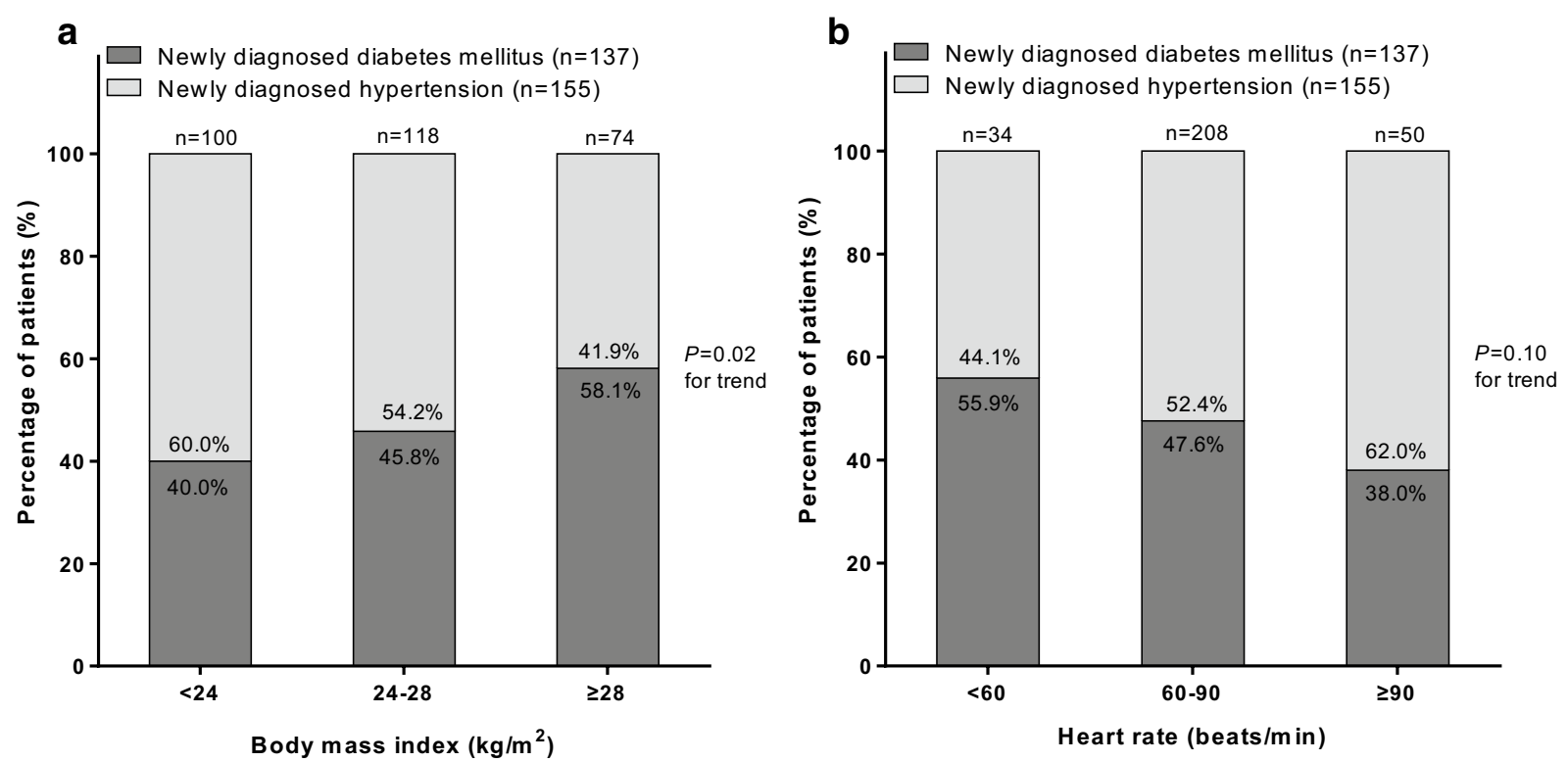

Fig. 1 Percentage of hypertensive patients with newly diagnosed diabetes mellitus (solid) or diabetic patients with newly diagnosed hypertension (open) according to body mass index (a) and heart rate (b). The number and percentage of patients and $P$ values for trend are given 


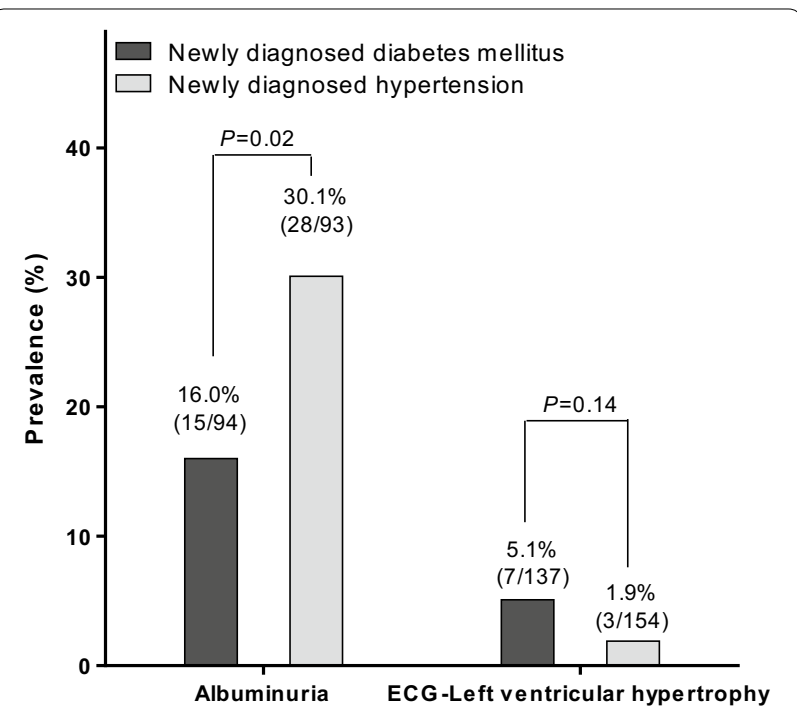

Fig. 2 Prevalence of albuminuria and electrocardiographic (ECG)-left ventricular hypertrophy in hypertensive patients with newly diagnosed diabetes mellitus (solid bar, $n=137$ ) or diabetic patients newly diagnosed hypertension (open bar, $n=155$ ). The number and percentage of patients and $P$ values are given

heart rate and lower prevalence of albuminuria than diabetic patients with newly diagnosed hypertension. Because the joint presence of these two clinical entities confers an even higher cardiovascular risk than either one alone, [2-4] our finding may have clinical implications in the prevention of this serious condition in those who already have either hypertension or diabetes mellitus alone.

Our observation on the risk of diabetes mellitus associated with a greater body mass index in patients with previously diagnosed hypertension is in keeping with the results of several previous prospective studies [1014]. In 15,089 treated hypertensive patients attending the Glasgow Blood Pressure Clinic, body mass index was a major predictor of new onset diabetes mellitus $(n=1862)$ immediately after blood glucose level at baseline during 40 years of follow-up [10]. Similar results were observed in a post hoc analysis on the predictors of new-onset diabetes mellitus in 9995 hypertensive nondiabetic patients enrolled in the Valsartan Antihypertensive Long Term Use Evaluation (VALUE) trial [11]. Again, body mass index was the second important predictor of new-onset diabetes mellitus $(n=1298)$ during a mean follow-up of 4.2 years. Although there is strong evidence on the link between obesity and diabetes mellitus in hypertensive patients, obesity can also be a marker of the pathophysiological pathway shared between hypertension and diabetes mellitus, such as the insulin resistance [15].
Our observation on the risk of newly diagnosed hypertension associated with a faster heart rate in patients with an established diagnosis of diabetes mellitus is also in line with the concept that resting heart rate is a marker of cardiovascular risk, as demonstrated in numerous observational studies [16-19]. Resting heart rate is a measure of sympathovagal balance [19]. Faster resting heart rate could behave as an indicator of the impairment in sympathovagal function associated with diabetes mellitus. It is therefore not necessarily the resting heart rate itself that confers the risk of hypertension. It is the sympathovagal imbalance that renders the diabetic patients to develop high blood pressure. There is at least one study that has reported significant association between resting heart rate and the incidence of hypertension during a mean follow-up of 3.5 years [20]. In this study, diabetes mellitus was adjusted, but not analyzed separately from those non-diabetic patients.

Our explanation on the higher prevalence of left ventricular hypertrophy in hypertensive patients with a newly diagnosed diabetes mellitus and higher prevalence of albuminuria in diabetic patients with a newly diagnosed hypertension can be straight forward. Left ventricular hypertrophy is a major cardiac structural complication of hypertension [21]. Patients with a longterm established hypertension therefore have a higher risk of left ventricular hypertrophy than those with a newly diagnosed hypertension. Albuminuria is a complication of both diabetes mellitus and hypertension [22]. That explains why in the present study the prevalence of albuminuria was much higher than that of left ventricular hypertrophy. However, the prevalence of albuminuria is high in the presence of diabetes mellitus only than in the presence of hypertension only [23, 24]. That explains why in the present study, diabetes mellitus with a newly diagnosed hypertension had a higher risk of albuminuria.

Our study should be interpreted within the context of its limitations. First, our study had a cross-section design and does not allow any causal inference. Although our patients had newly diagnosed hypertension or diabetes, our study was still different from a prospective observational one. Second, our study had a relatively small sample size with few measurements of biological markers. Third, albuminuria was evaluated on a single spot urine sampling. However, albuminuria and creatinine were measured in a core laboratory. A stringent quality assurance programme was implemented, including the exclusion of patients with suspected urinary tract infections. Fourth, we did not collect information on the frequency of patients' clinic visit. Hypertensive and diabetic patients in China are usually followed up on a monthly basis for drug prescriptions. However, it is still possible that the time interval varied between patients, which might influence the between-group comparisons. 


\section{Conclusion}

In conclusion, earlier or later onset of hypertension than diabetes mellitus may have different risk predictors and organ damages. Our finding should be tested in prospective intervention studies on the possible pathophysiological pathways behind obesity and tachycardia.

\section{Supplementary information}

Supplementary information accompanies this paper at https://doi. org/10.1186/s13098-019-0465-3.

Additional file 1: Appendix 1. Participating hospitals of the China ATTEND Registry.

\section{Abbreviations}

HbA1c: glycosylated haemoglobin A1; OGTT: oral glucose tolerance test; ECG: electrocardiogram; LDL: low-density lipoprotein; HDL: high-density lipoprotein; BMl: body mass index.

\section{Acknowledgements}

The authors gratefully acknowledge the participation of the patients and the contribution of the investigators. The participating hospitals were listed in an Additional file 1: Appendix 1 (http://links.lww.com/HJH/A634).

\section{Authors' contributions}

All authors participated in the development of the analysis plan, interpretation of the results, and in reviewing and editing the manuscript. All authors read and approved the final manuscript.

\section{Funding}

The registry was sponsored by Sanofi China (Shanghai) (DIREG_L_05728).

\section{Availability of data and materials}

Qualified researchers may request access to patient level data and related study documents including the clinical study report, study protocol with any amendments, blank case report forms, statistical analysis plan, and dataset specifications. Patient level data will be anonymized and study documents will be redacted to protect the privacy of our study participants. Further details on Sanofi's data sharing criteria, eligible studies, and process for requesting access can be found at: https://www.clinicalstudydatarequest.com/.

\section{Ethics approval and consent to participate}

The ethics committees of all participating hospitals approved the study protocol. All subjects gave written informed consent.

\section{Consent for publication}

Not applicable.

\section{Competing interests}

The authors declare that they have no competing interests.

\section{Author details}

${ }^{1}$ Centre for Epidemiological Studies and Clinical Trials, Shanghai Key Laboratory of Hypertension, The Shanghai Institute of Hypertension, Department of Hypertension, Ruijin Hospital, Shanghai Jiaotong University School of Medicine, Ruijin 2nd Road 197, Shanghai 200025, China. ${ }^{2}$ Department of Endocrinology, Renmin Hospital, Peking University, Beijing, China.

Received: 7 June 2019 Accepted: 14 August 2019

Published online: 23 August 2019

\section{References}

1. Climie RE, van Sloten TT, Bruno RM, Taddei S, Empana JP, Stehouwer CDA, Sharman JE, Boutouyrie P, Laurent S. Macrovasculature and microvasculature at the crossroads between type 2 diabetes mellitus and hypertension. Hypertension. 2019;73:1138-49.

2. Sehestedt T, Hansen TW, Li Y, Richart T, Boggia J, Kikuya M, Thijs L, StolarzSkrzypek K, Casiglia E, Tikhonoff V, Malyutina S, Nikitin Y, BjörklundBodegård K, Kuznetsova T, Ohkubo T, Lind L, Torp-Pedersen C, Jeppesen J, Ibsen H, Imai Y, Wang J, Sandoya E, Kawecka-Jaszcz K, Staessen JA, on behalf of the International Database on Ambulatory blood pressure in relation to Cardiovascular Outcomes (IDACO) Investigators. Are blood pressure and diabetes additive or synergistic risk factors? Outcome in 8494 subjects randomly recruited from 10 populations. Hypertens Res. 2011;34:714-21.

3. Long AN, Dagogo-Jack S. Comorbidities of diabetes and hypertension: mechanisms and approach to target organ protection. J Clin Hypertens. 2011:13:244-51.

4. Chen G, McAlister FA, Walker RL, Hemmelgarn BR, Campbell NR. Cardiovascular outcomes in Framingham participants with diabetes: the importance of blood pressure. Hypertension. 2011;57:891-7.

5. Song J, Sheng CS, Huang QF, Li LH, Ma CS, Guo XH, Ji LN, Wang JG. Management of hypertension and diabetes mellitus by cardiovascular and endocrine physicians: a China registry. J Hypertens. 2016;34:1648-53.

6. Mancia G, Grassi G, Zanchetti A. New-onset diabetes and antihypertensive drugs. J Hypertens. 2006;24:3-10.

7. Zheng SL, Roddick AJ, Aghar-Jaffar R, Shun-Shin MJ, Francis D, Oliver N, Meeran K. Association between use of sodium-glucose cotransporter 2 inhibitors, glucagon-like peptide 1 agonists, and dipeptidyl peptidase 4 inhibitors with all-cause mortality in patients with type 2 diabetes. A systematic review and meta-analysis. JAMA. 2018;319:1580-91.

8. Williams B, Mancia G, Spiering W, Aboyans V, Desormais I, ESC Scientific Document Group. ESC/ESH Guidelines for the management of arterial hypertension. Eur Heart J. 2018;2018(39):3021-104.

9. Joint committee for guideline revision. 2016 Chinese guidelines for the management of dyslipidemia in adults. J Geriatr Cardiol. 2018;15:1-29.

10. Lip S, Jeemon P, McCallum L, Dominiczak AF, Mclnnes GT, Padmanabhan S. Contrasting mortality risks among subgroups of treated hypertensive patients developing new-onset diabetes. Eur Heart J. 2016:37:968-74.

11. Aksnes TA, Kjeldsen SE, Rostrup M, Storset O, Hua TA, Julius S. Predictors of new-onset diabetes mellitus in hypertensive patients: the VALUE trial. J Hum Hypertens. 2008;22:520-7.

12. Hsia DS, Larrivee S, Cefalu WT, Johnson WD. Impact of lowering BMI cut points as recommended in the revised American Diabetes Association's standards of medical care in diabetes - 2015 on diabetes screening in Asian Americans. Diabetes Care. 2015;38:2166-8.

13. Bakris G, Stockert J, Molitch M, Zhou Q, Champion A, Bacher P, Sowers J. Risk factor assessment for new onset diabetes: literature review. Diabetes Obes Metab. 2009;11:177-87.

14. Wing RR, Blair EH, Bononi P, Marcus MD, Watanabe R, Bergman RN. Caloric restriction per se is a significant factor in improvements in glycemic control and insulin sensitivity during weight loss in obese NIDDM patients. Diabetes Care. 1994;17:30-6.

15. Lastra G, Syed S, Kurukulasuriya LR, Manrique C, Sowers JR. Type 2 diabetes mellitus and hypertension: an update. Endocrinol Metab Clin North Am. 2014;43:103-22.

16. Fox K, Borer JS, Camm AJ, Danchin N, Ferrari R, Lopez Sendon JL, Steg PG, Tardif JC, Tavazzi L, Tendera M. Resting heart rate in cardiovascular disease. JACC. 2007;50:823-30.

17. Palatini P, Rosei EA, Casiglia E, Chalmers J, Ferrari R, Grassi G, Inoue T, Jelakovic B, Jensen MT, Julius S, Kjeldsen SE, Mancia G, Parati G, Pauletto P, Stella A, Zanchetti A. Management of the hypertensive patient with elevated heart rate: Statement of the Second Consensus Conference endorsed by the European Society of Hypertension. J Hypertens. 2016;34:813-21.

18. Hillis GS, Woodward M, Rodgers A, Chow CK, Li Q, Zoungas S, Patel A, Webster R, Batty GD, Ninomiya T, Mancia G, Poulter NR, Chalmers J. Resting heart rate and the risk of death and cardiovascular complications in patients with type 2 diabetes mellitus. Diabetologia. 2012;55:1283-90.

19. Hillis GS, Hata J, Woodward M, Perkovic V, Arima H, Chow CK, Zoungas S, Patel A, Poulter NR, Mancia G, Williams B, Chalmers J. Resting heart rate and the risk of microvascular complications in patients with type 2 diabetes mellitus. JAHA. 2012;1:e002832. 
20. Wang A, Liu X, Guo X, Dong Y, Wu Y, Huang Z, Xing A, Luo Y, Jonas JB, Wu S. Resting heart rate and risk of hypertension: results of the Kailuan cohort study. J Hypertens. 2014;32:1600-5.

21. Soliman EZ, Byington RP, Bigger JT, Evans G, Okin PM, Goff DC Jr, Chen H. Effect of intensive blood pressure lowering on left ventricular hypertrophy in patients with diabetes mellitus: action to Control Cardiovascular Risk in Diabetes Blood Pressure Trial. Hypertension. 2015;66:1123-9.

22. Shin DI, Seung KB, Yoon HE, Hwang BH, Seo SM, Shin SJ, Kim PJ, Chang $\mathrm{K}$, Baek SH. Microalbuminuria is independently associated with arterial stiffness and vascular inflammation but not with carotid intima-media thickness in patients with newly diagnosed type 2 diabetes or essential hypertension. J Korean Med Sci. 2013;28:252-60.

23. Menne J, Izzo JL Jr, Ito S, Januszewicz A, Katayama S, Chatzykirkou C, Mimran A, Rabelink TJ, Ritz E, Ruilope LM, Rump LC, Viberti G, Haller H.
Prevention of microalbuminuria in patients with type 2 diabetes and hypertension. J Hypertens. 2012;30:811-8.

24. Bruno RM, Penno G, Daniele G, Pucci L, Lucchesi D, Stea F, Landini L, Cartoni G, Taddei S, Ghiadoni L, Del Prato S. Type 2 diabetes mellitus worsens arterial stiffness in hypertensive patients through endothelial dysfunction. Diabetologia. 2012;55:1847-55.

\section{Publisher's Note}

Springer Nature remains neutral with regard to jurisdictional claims in published maps and institutional affiliations.
Ready to submit your research? Choose BMC and benefit from:

- fast, convenient online submission

- thorough peer review by experienced researchers in your field

- rapid publication on acceptance

- support for research data, including large and complex data types

- gold Open Access which fosters wider collaboration and increased citations

- maximum visibility for your research: over $100 \mathrm{M}$ website views per year

At BMC, research is always in progress.

Learn more biomedcentral.com/submissions 